\title{
Umgang mit Marktunsicherheiten in der Zielsystementwicklung: Methode zur Reduktion von \\ Definitionslücken bei der
}

\section{Konkretisierung des Initialen Zielsystems}

Valentin Zimmermann, Christoph Kempf, Leo Hartmann, Nikola Bursac, Albert Albers

Der systematische Umgang mit Unsicherheiten, die in Form von Wissens- und Definitionslücken vorliegen, stellt eine zentrale Aktivität der Produktentwicklung dar. Im Zuge der Zielsystementwicklung liegen Unsicherheiten insbesondere in Form von aus Kunden- und Anwendersicht nichtzutreffender und fehlender oder unvollständiger Ziele und Anforderungen vor. Um bei der Konkretisierung des initialen Zielsystems dahingehend zu unterstützen, wurde eine Methode abgeleitet, welche die systematische Integration von Kunden und Anwendern in die Erhebung von Zielsystemelementen adressiert. Dabei formulieren Kunden und Anwender gemeinsam mit Produktentwicklern Ziele für das zu entwickelnde Produkt. Um dies zu unterstützen, werden die Ziele in Form von Satzschablonen formuliert, um die Vollständigkeit der Ziele zu gewährleisten. Weiter kann durch den Aufbau der Satzschablone sichergestellt werden, dass die Begründung in Form des Kunden-oder Anwendernutzens dokumentiert ist. Zusätzlich wurde ein Portfolio abgeleitet, welches die Ziele entsprechend der Zielgruppe und des relevanten Use-Cases strukturiert und damit fehlende Ziele darlegt. Im Rahmen einer Evaluation konnte gezeigt werden, dass durch die Anwendung der Methode in einem Entwicklungsprojekt von Hekatron Brandschutz die Vollständigkeit des Zielsystems gesteigert und die vorliegende Unsicherheit reduziert werden konnte.

Keywords: Zielsystem, Produktgenerationsentwicklung, Kundenintegration, Anforderungsmanagement, Unsicherheit.

\section{Einleitung}

Das Streben nach Innovation (erfolgreiche Produkte) und damit Markterfolg, treibt Unternehmen seit jeher als Motivator in der Produktentwicklung (Ili, Albers, Miller, 2010, S. 255). Neben einer guten Idee und einer technischen und/oder serviceseitigen Lösung, der Invention, und der erfolgreichen Einführung am Markt, ist allerdings auch 
ein relevantes Produktprofil für Markterfolg und damit eine Innovation erforderlich. Dieses Produktprofil beschreibt dabei die Bedarfssituation am Markt (Albers et al., 2018, S.6).

Da das Streben nach neuen technischen Lösungen, also Inventionen, die natürliche Veranlagung vieler Entwickler zu sein scheint, fällt die Spezifikation von technischen Anforderungen als Entwicklungsziele meist nicht schwer. Anders sieht es jedoch mit der Ermittlung und Berücksichtigung der Bedarfssituation aus. Wie oben beschrieben, ist Kenntnis dieser, jedoch notwendige Voraussetzung für Innovation. Dies macht es folglich notwendig auch die Kunden- und Anwendersicht in die Erhebung von Zielsystemelementen zu integrieren. Die Verbesserung dieser Integration, sowie die Erhöhung des Verständnisses von Entwicklern für die Notwendigkeit der Kunden- und Anwendersicht schon in der Anforderungserhebung ist das Ziel dieser Veröffentlichung.

\section{Stand der Forschung}

\section{Zielsystemmanagement im Modell der PGE - Produktgenerationsentwicklung}

In der Produktentwicklung gilt es ein vages Zielsystem durch ein Handlungssystem in ein konkretes Objektsystem zu überführen. Das Zielsystem enthält dabei die Ziele und Randbedingungen sowie deren Wechselwirkungen und Begründungen, um das richtige Produkt zu entwickeln. (Albers, 2010, S.4) Wichtiger Ausgangspunkt ist hierbei die Kenntnis der Bedürfnisse und Interessen der künftigen Kunden und Anwender. Durch die frühzeitige Kenntnis dieser Bedürfnisse kann die Produktentwicklung daran ausgerichtet und dagegen validiert werden. AlBERS bietet mit dem Produktprofil einen Ausgangspunkt für die Produktentwicklung, indem dieses den angestrebten Kunden-, Anwender- sowie den Anbieternutzen beschreibt. (Albers et al., 2018, S. 3) Neben dem Produkt selbst, enthält das Objektsystem alle im Produktentstehungsprozess entstehende Artefakte. Verbunden sind Ziel- und Objektsystem über das Handlungssystem, welches die Ressourcen, Methoden und Prozesse bereitstellt (Albers, 2010, S.5). Zielund Objektsystem werden dabei durch einen Wechsel von Synthese- und Analyseaktivitäten erstellt (Albers, Ebel \& Lohmeyer, 2012).

Nach Albers kann dabei die Entwicklung von neuen Produkten durch das Modell der PGE - Produktgenerationsentwicklung beschrieben werden (Albers, Bursac \& Wintergerst, 2015). Diesem Verständnis entsprechend kann die Entwicklung von neuen Produkten als die Entwicklung neuer Produktgenerationen $\left(G_{n}\right.$ als aktuell in der Entwicklung befindliche Produktgeneration) auf Basis eines Referenzsystems $R_{n}$ verstanden 
werden (vgl. Abbildung 1). Referenzsystemelemente können dabei sowohl Vorgänger( $G_{n-1}$ als direkte Vorgängergeneration der $G_{n}$ am Markt) als auch Wettbewerbsprodukte bzw. deren Teilsysteme sein. Während dem Entwicklungsprozess wird das Referenzsystem kontinuierlich weiterentwickelt. (Albers et al., 2019, S.1699) Die neue Produktgeneration wird ausgehend von den im Referenzsystem beschriebenen Referenzsystemelementen durch die Aktivitäten der Übernahme-, Gestalt- und Produktvariation entwickelt (Albers, Bursac \& Wintergerst, 2015, S.18). Durch die Nutzung eines Referenzsystems als Startpunkt, unterstützt das Modell der PGE die Nutzung existierenden Wissens für die Entwicklung neuer Produktgenerationen.

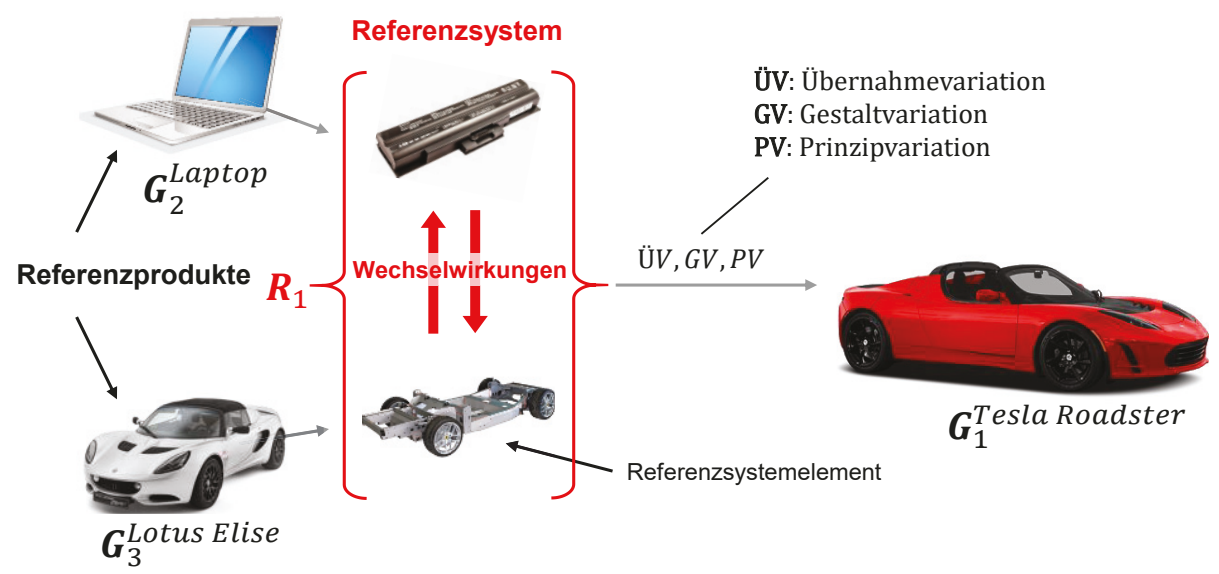

Abbildung 1: Vereinfachte Darstellung des Modells der PGE angelehnt an (Albers et al., 2019, S.1699)

\section{Marktunsicherheiten im Zielsystemmanagement}

Im Rahmen einer Literatur- und Interviewstudie haben ZIMMERMANN et al. (Zimmermann, Heimicke, Bohn, Schuhmacher, Trost \& Albers, 2020) identifiziert, dass im Zuge der Zielsystementstehung insbesondere ein adäquater Umgang mit Marktunsicherheiten, von Bedeutung ist. Allerdings besteht eine Vielzahl an Herausforderungen hinsichtlich dieses Umgangs. Marktunsicherheiten lassen sich, entsprechend dem Verständnis von MCMANUS und HASTINGS (2004, S.84) durch vorliegende Wissens- (fehlendes Wissen) und Definitionslücken (ausstehende Entscheidungen und Spezifikationen) beschreiben und umfassen somit beispielsweise entsprechende Lücken hinsichtlich der Kundenbedürfnisse (Logue \& McDaid, 2008, S.73), des Anwendungsfalls oder der Zahlungsbereitschaft (Verworn \& Herstatt, 2007, S.115). Um die Produktentwickler im 
Zuge der Zielsystementwicklung beim Umgang mit Marktunsicherheiten zu unterstützen, leiten ZIMmERMANN et al. (Zimmermann, Heimicke, Bohn, Schuhmacher, Trost \& Albers, 2020, S.255) vier konsekutiv durchführbare Methoden ab. Dabei wird zunächst im Rahmen der Methode Initiales Zielsystem ableiten aufbauend auf einer Analyse der bereits bekannten Referenzsystemelemente durch eine Kunden- und Anwenderbefragung die zu differenzierenden, erlebbaren Produkteigenschaften der Produktgeneration $\mathrm{G}_{\mathrm{n}}$ abgeleitet (Albers, Zimmermann, Marthaler, Bursac, Duehr \& Spadinger, 2021 in Druck). In der sich anschließende Methode Initiales Zielsystem konkretisieren, die Bestandteil dieser Veröffentlichung ist, gilt es geeignete Zielsystemelemente zu erheben. Im Rahmen der dritten Methode Initiales Zielsystem bewerten werden die identifizierten Zielsystemelemente systematisch hinsichtlich der vorliegenden Marktunsicherheiten bewertet (Zimmermann, Prinz \& Albers, 2020). Abschließend werden im Rahmen der Methode Initiales Zielsystem validieren Minimum Viable Products (MVPs) ausgewählt und eine Validierung mit Kunden und Anwendern durchgeführt, um systematisch Marktunsicherheiten zu reduzieren.

In Kontext der Konkretisierung des initialen Zielsystems werden Methoden zur Integration der Bedürfnisse von Anwender und Kunde in den Prozess der Anforderungserhebung diskutiert. Diese sollen dabei unterstützten, die aus deren Sicht richtigen Zielsystemelemente zu erfassen und damit die vorliegenden Wissenslücken zu reduzieren. Neben Ansätzen, wie Kundeninterviews (Dieste, Juristo \& Schull, 2008) oder Lead-User Ansätzen (Herstatt \& Hippel, 1992) werden insbesondere Kreativitätsmethoden, wie beispielsweise die Persona-Methode (u.a. Nielsen, 2018; Pruitt \& Grudin, 2003) dargelegt. Im Zuge dieser Methode werden fiktive Personen auf Basis von Charaktereigenschaften und Anforderungen beschrieben und in den Produktentwicklungsprozess integriert. Somit ermöglichen sie eine verstärkte Ausrichtung auf die Bedürfnisse der Zielgruppe. Andere Ansätze unterstützen das Strukturieren und Explizieren von Wissen. Durch Use-Case Diagramme der Systems Modeling Language (SysML) (INCOSE, 2015) wird eine Verknüpfung zwischen den Elementen Anforderungen, Anwendungsfällen und Stakeholdern hergestellt und damit Definitionslücken in Form fehlender Elemente oder Verknüpfungen adressiert. Story Cards (Bik, Lucassen \& Brinkkemper, 2017), welche eine aus den Elementen Stakeholder, Use-Case und Nutzen bestehende Satzschablone für die Formulierung von Zielen und Anforderungen sind, adressieren ebenfalls diesen Aspekt. 


\section{Forschungsdesign}

Ein adäquater Umgang mit Marktunsicherheiten in der Zielsystementwicklung ist unabdingbar, um den Erfolg eines Produktentwicklungsvorhabens sicherzustellen. Dabei gilt es bestehende Wissens- und Definitionslücken hinsichtlich der Bedürfnisse von Kunden und Anwender bereits in einer frühen Phase zu schließen. Dabei ist neben einer geeigneten Auswahl der zu differenzierenden Produkteigenschaften, welche den Neuentwicklungsanteil maßgeblich bestimmen, der Identifikation der mit Unsicherheiten behafteten Zielsystemelemente und Validierung dieser, insbesondere die Erhebung von Zielsystemelementen von Relevanz. Bei dieser Konkretisierung des initialen Zielsystems ist neben der Identifikation, der aus Kunden- und Anwendersicht richtigen Ziele und damit der Reduktion von Wissenslücken, insbesondere die Gewährleistung eines angemessenen Maßes an Vollständigkeit von Relevanz. Die Vollständigkeit bezieht sich dabei einerseits auf die Summe der erhobenen Ziele, aber andererseits auch darauf, dass bei den erhobenen Zielen keine notwendigen Informationen fehlen und addressiert damit die Reduktion von Definitionslücken. In der Literatur werden verschiedene Ansätze der Integration der Kunden- und Anwenderbedürfnisse in die Zielsystemkonkretisierung diskutiert, die dabei unterstützen, die richtigen Zielsystemelemente zu erheben und damit Wissenslücken zu schließen. Darüber hinaus bestehen Ansätze zum Strukturieren von Wissen, welche dazu dienen Definitionslücken zu identifizieren und damit diese zu schließen. Zielsetzung dieser Arbeit ist es, durch die Kombination verschiedener bestehender methodischer Elemente den Prozess der Konkretisierung des initialen Zielsystems bei der Integration von Kunden und Anwender dahingehend zu unterstützen, ausreichend „richtige“ Zielsystemelemente zu erheben, um somit gezielt Wissens- und insbesondere Definitionslücken zu schließen und damit Marktunsicherheiten zu reduzieren. Dazu werden die folgenden Forschungsfragen beantwortet:

1. Welche Handlungspotentiale bestehen beim Erheben von Zielsystemelementen im anlagentechnischen Brandschutz hinsichtlich des Umgangs mit Marktunsicherheiten entsprechend der Einschätzung der Entwickler?

2. Wie ist eine methodische Unterstützung zur Integration von Kunden und Anwendern in den Prozess der Zielsystemkonkretisierung zu konzipieren, mit dem Ziel Definitionslücken zu schließen und damit Marktunsicherheiten zu reduzieren?

3. Welchen Beitrag leistet die Methode bei der Reduktion, der in der Form von Wissens- und Definitionslücken vorliegenden Marktunsicherheiten? 
Die im Rahmen dieser Veröffentlichung durchgeführte Forschung wurde entsprechend der Design Research Methodology nach BLESSING und CHAKRABARTI (2009) in drei aufeinander aufbauende Studien gegliedert. Im Zuge der Deskriptiven Studie 1 wurden durch eine Interview- und Fragebogenstudie mit fünf Mitarbeitern von Hekatron Brandschutz Handlungspotentiale beim Erheben von Anforderungen erfasst. Im Anschluss wurde im Rahmen der Präskriptiven Studie ein Ansatz abgeleitet, der Produktentwickler dabei unterstützt, gezielt Kunden und Anwender in den Prozess der Konkretisierung des initialen Zielsystems zu integrieren und deren Bedürfnisse zu erfassen. Abschließend wurde im Rahmen der Deskriptiven Studie 2 evaluiert, welchen Beitrag der entwickelte Ansatz im Umfeld von Hekatron Brandschutz, hinsichtlich der Reduktion der vorliegenden Marktunsicherheiten in Form von Wissens- und Definitionslücken, leistet. Dazu bewerteten 15 Experten des anlagentechnischen Brandschutzes fragebogenbasiert die vorliegende Entwicklungssituation hinsichtlich der vorliegenden Marktunsicherheit.

\section{Handlungspotentiale beim Erheben von Zielen und Anforderungen}

Auf Basis einer Literaturrecherche wurden Herausforderungen identifiziert, welche im Zuge der Konkretisierung von initialen Zielsystemen auftreten. Diese wurden durch Produktentwickler von Hekatron Brandschutz hinsichtlich der Relevanz bewertet (vgl. Abbildung 2). Die, aus Sicht der Produktentwickler relevanteste Herausforderung, neben der Identifikation der aus Kunden- und Anwendersicht richtigen Zielsystemelemente, ist der Umgang mit aus Kunden- und Anwendersicht fehlenden Zielsystemelementen. Hinsichtlich dieser fehlenden Zielsystemelemente führen die Probanden im Rahmen der Interviews aus, dass neben fehlenden Zielen, welche Kunden und Anwender hinsichtlich des Produktes bzw. im Umgang mit diesem haben, insbesondere auch fehlende Rahmenbedingungen, die sich beispielsweise aus der (beim Kunden) vorhanden Infrastruktur ergeben, kritisch für die Produktentwicklung sind.

Nach Ansicht der Probanden sind weitere relevante Herausforderungen, dass Zielsystemelemente teilweise unvollständig sind bzw. den in Prosa formulierten Zielsystemelementen häufig wichtige Informationen, wie beispielsweise der zugehörige Kundenoder Anwendernutzen, oder quantitative Angaben fehlen. Dies führt nach Ansicht der befragten Probanden häufig dazu, dass aus Kunden- und Anwendersicht das falsche Produkt entwickelt wird.

Hinsichtlich der Potentiale, welche die Probanden sehen, gilt es vor allem die Integration von bestehendem Wissen in die Erhebung von Zielsystemelementen zu erwähnen. 
So stellen die Probanden insbesondere die Chance heraus auf Basis von Use-Cases zielgerichtet Zielsystemelemente ableiten zu können. Dabei lassen sich diese UseCases, nach Ansicht der Probanden, durch bereits im Markt verfügbare Produktgenerationen und die im Feld verfügbare Infrastruktur identifizieren. Ein weiteres Potential, das die befragten Probanden sehen, ist die gezielte Integration der Kunden und Anwender beim Erheben von Zielsystemelementen. Es wird angenommen, dass durch diese Integration, sowohl die Entwicklungsdauer, als auch die Kunden- und Anwenderzufriedenheit mit dem entwickelten Produkt und damit auch der Produkterfolg am Markt positiv beeinflusst werden kann. Allerdings führen die Probanden auf Nachfrage aus, dass diese Potentiale aktuell nur unzureichend genutzt werden. Ursachen sehen die befragten Probanden insbesondere darin, dass Schwierigkeiten in der Kommunikation zwischen Entwicklern auf der einen und Kunden und Anwender auf der anderen Seite bestehen. So beschreiben Kunden bzw. Anwender häufig eine Lösung statt des Bedarfes, sodass den Entwicklern, welche ebenfalls vorrangig in Lösungen denken, häufig nicht klar ist, warum entsprechende Anforderungen umzusetzen sind. Weiter fällt es oft schwer, die Bedürfnisse in Zielsystemelemente zu überführen, welche das Produkt aus technischer Sicht beschreiben.

Herausforderung

Aus Kunden- und Anwendersicht fehlende Zielsystemelemente (ZSE) Identifikation der aus Kundenund Anwendersicht richtigen ZSE

Unvollständigkeit der ZSE

Mehrdeutigkeit der ZSE

Ausreichende Integration von Kunden und Anwendern Überspezifikation von ZSE

Widersprüche und Redundanzen zwischen ZSE

Lösungsneutralität der ZSE

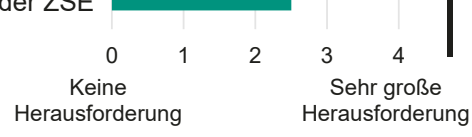

\section{Potential Kunden- und Anwenderintegration}

Sehr großer Einfluss
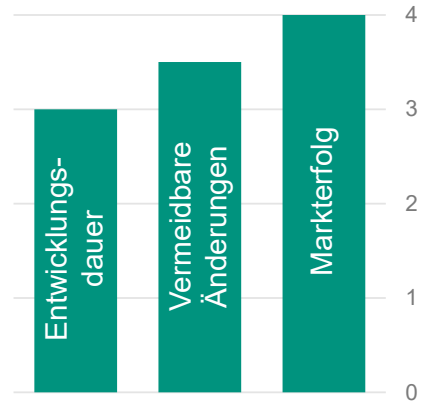

Kein Einfluss

Abbildung 3: Links: Relevanz verschiedener Herausforderungen.

Rechts: Potential der Kunden und Anwenderintegration. 


\section{Methode zum Erheben von Zielsystemelementen}

Auf Basis der Ergebnisse der Deskriptiven Studie 1 lässt sich die Zielsetzung ableiten, Produktentwickler systematisch beim Erheben von Zielsystemelementen hinsichtlich der Integration von Kunden und Anwendern und dem mit den relevanten Referenzsystemelementen verknüpften Wissen zu unterstützen, um kontinuierlich Marktunsicherheiten zu reduzieren. Diese Marktunsicherheit prägt sich primär in Definitionslücken (fehlende Zielsystemelemente und unzureichender Vollständigkeit der Zielsystemelemente) und Wissenslücken (aus Kunden- und Anwendersicht falsche Zielsystemelemente) aus.

Im Vorfeld der Methodendurchführung, werden die ausgewählten Referenzsystemelemente systematisch durch Kunden und Anwender bewertet (Albers, Zimmermann, Marthaler, Bursac, Duehr \& Spadinger, 2021 in Druck). Die dabei gesammelten Informationen, wie die aus Kunden- und Anwendersicht zu differenzierende erlebbare Produkteigenschaften, werden in Form von Personas bzw. deren Anforderungen an das zu entwickelnde Produkt aufbereitet und gezielt in die Zielsystemkonkretisierung integriert. Zusätzlich werden die Referenzsystemelemente mit Fokus auf die bereits im Markt verfügbare Produktgeneration $G_{n-1}$ und die bereits vorhandene Systemumgebung hinsichtlich der relevanten Use-Cases analysiert. Dies schränkt folglich einerseits den zu betrachtenden Lösungsraum ein, andererseits kann damit die Kreativität gezielt aktiviert werden bzw. zusätzliche, bisher nicht betrachtete Handlungsfelder dargelegt werden. Um dies zu systematisieren, wird die Zielsystemkonkretisierung entsprechend eines Portfolios strukturiert, welches in Anlehnung an Use-Case Diagramme der System Modeling Language gestaltet ist (vgl. Abbildung 3). In dem Portfolio werden auf der horizontalen Achse sämtliche relevante Use-Cases entsprechend der zugehörigen Produktlebenszyklusphase abgebildet. Auf der Vertikalen werden die relevanten Stakeholder - Kunde und Anwender - dargestellt, wobei diese durch die erstellten Personas weiter detailliert werden können. Im Rahmen der Zielsystemkonkretisierung werden nun Zielsystemelemente entsprechend der verschiedenen Portfoliofelder formuliert. Dabei unterstützt das Portfolio gezielt die Formulierung der Zielsystemelemente aus Sicht der verschiedenen Stakeholder und zeigt systematisch Definitionslücken auf. 


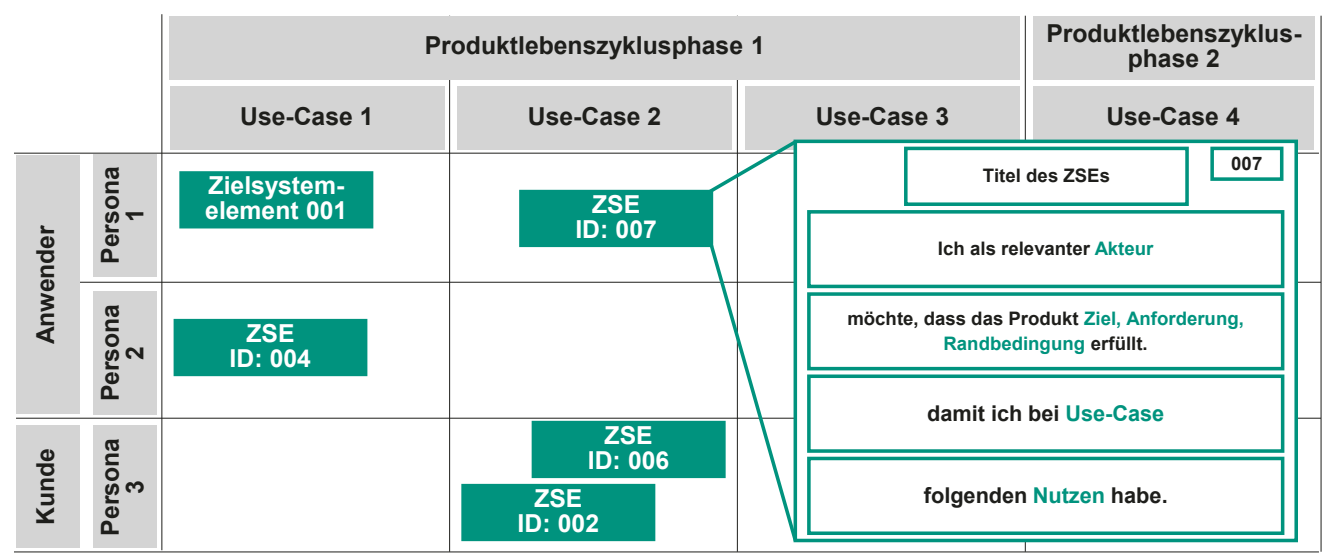

Abbildung 4: Portfolio zur Strukturierung des Zielsystems

Im Rahmen der Methodendurchführung wird ein mehrstufiger Brainwritingspool (Heslin, 2009) durchgeführt, an dem sowohl Entwickler als auch Kunden und Anwender teilnehmen, wobei in jeder Stufe neue Impulse (Use-Cases, Personas etc.) integriert werden. Dabei formulieren die Teilnehmer Zielsystemelemente für das Produkt in Form von Satzschablonen, die entsprechend der Struktur von StoryCards aufgebaut sind. Somit kann sichergestellt werden, dass der Anwender- bzw. Kundennutzen, der durch das Zielsystemelement adressiert ist, auch expliziert wird, was wiederum einen Zugang zur Validierung darstellt. Darüber hinaus kann durch das Verwenden der Satzschablone gewährleistet werden, dass sämtliche relevanten Informationen, insbesondere die Begründung der Zielsystemelemente einheitlich dokumentiert sind und stellt damit einen weiteren Stellhebel zum Umgang mit Definitionslücken dar.

\section{Evaluation in einem realen Entwicklungsprojekt}

Zur Evaluation der Methode, wurde diese im Rahmen eines realen Produktentwicklungsprojektes bei Hekatron Brandschutz angewendet, in welchem die neue Produktgeneration eines Prüfgerätes für Brandmelder entwickelt wurde. Dazu wurden im Vorfeld bereits die zugehörigen Referenzsystemelemente ausgewählt und darauf aufbauend die relevanten Personas und Use-Cases abgeleitet. Im Anschluss wurde ein Workshop durchgeführt, wobei neben einem Moderator, drei Produktentwickler und zwei Vertriebsmitarbeiter bzw. Mitarbeiter des technischen Kundendiensts als Vertreter der 
Kunden und Anwender involviert waren, da aufgrund der vorherrschenden Restriktionen verbunden mit der Covid-19 Pandemie keine Kunden oder Anwender teilnehmen konnten.

Fünfzehn Experten des anlagentechnischen Brandschutzes bewerteten quantitativ die beiden Zielgrößen vorliegende Sicherheit, dass die Zielsystemente das aus Kundenund Anwendersicht richtige Produkt beschreiben und Vollständigkeit des Zielsystems und der Zielsystemelemente. Die vorliegende Sicherheit bildet dabei ein Indikator hinsichtlich der Wissenslücken und die Vollständigkeit ein Indikator hinsichtlich vorliegender Definitionslücken, wobei im Rahmen der Befragung explizit herausgestellt wurde, dass die Bewertung nicht absolut zu erachten ist, sondern vielmehr relativ, da das Zielsystem zu diesem Zeitpunkt per Definition nicht vollständig sein kann. Die Bewertung erfolgt sowohl vor, als auch nach der Methodendurchführung, wobei die Veränderung der Zielgröße relevanter ist, als die absoluten Werte. Zusätzlich erfolgte nach der Methodendurchführung eine qualitative Bewertung der Veränderung dieser Zielgrößen.
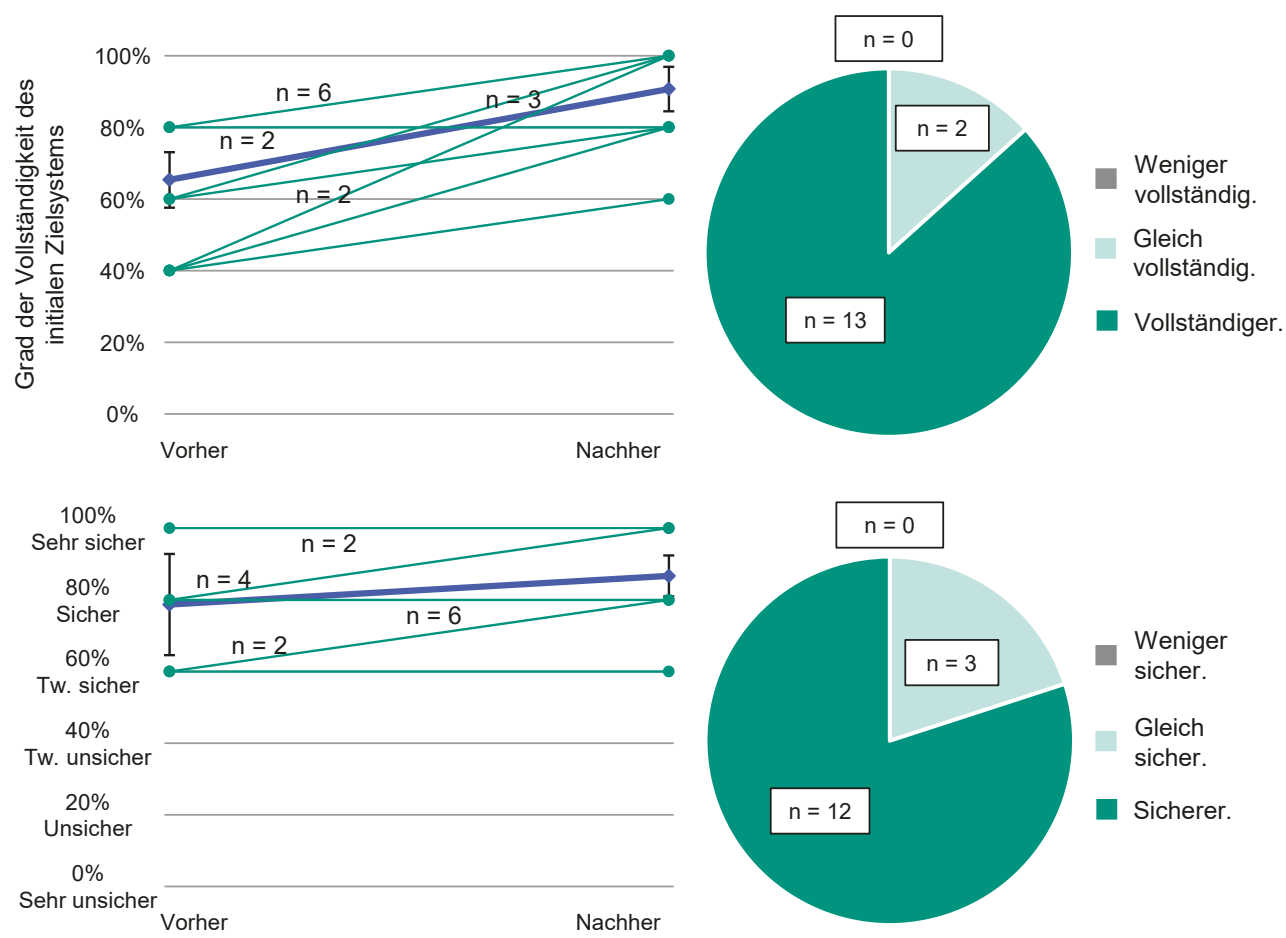
Abbildung 5: Oben: Quantitative und qualitative Bewertung der Vollständigkeit; unten: Quantitative und qualitative Bewertung der Sicherheit

Im Rahmen der quantitativen Bewertung der Vollständigkeit ergibt sich ein Anstieg von 65,3 \% auf 90,7 \%. Sämtliche der Experten schätzen den Grad der Vollständigkeit nach der Methodendurchführung gleich oder höher ein als vor der Durchführung dieser. Dies wird auch in der qualitativen Bewertung ersichtlich. Hier geben 13 der befragten Experten an, dass der Grad der Vollständigkeit zugenommen hat, lediglich zwei Experten nehmen den gleichen Grad wahr.

Auch bei der quantitativen Bewertung der vorliegenden Sicherheit ist ein Anstieg $(78,7 \%$ auf $86,7 \%)$ zu erkennen, wobei dieser nicht so deutlich ausfällt wie bei der Bewertung der Vollständigkeit. Dies zeigt sich auch in der qualitativen Bewertung. Hier geben zwölf Probanden an, nach der Methodendurchführung sicherer zu sein, dass das durch die Zielsystemelemente beschriebene Produkt die Bedürfnisse der Kunden und Anwender erfüllt und drei Probanden empfinden die gleiche Sicherheit bzw. Unsicherheit.

\section{Diskussion und Ausblick}

Nach Ansicht der Probanden belaufen sich die zentralen Herausforderungen der Zielsystemkonkretisierung neben dem Erheben der richtigen Zielsystemelemente, insbesondere auf die Reduktion von Definitionslücken, welche sich einerseits in Form fehlender Zielsystemelemente und andererseits in Form fehlender Informationen der Zielsystemelemente kenntlich machen. Einen relevanten Stellhebel sehen die befragten Probanden insbesondere in der Integration von Kunden und Anwendern in den Prozess der Erhebung von Zielsystemelementen. Durch die gezielte Kombination methodischer Elemente, wie Personas oder Story-Cards zur Unterstützung der gemeinsam mit Kunden und Anwendern durchgeführten Zielsystemkonkretisierung, können systematisch Marktunsicherheiten reduziert werden. So wird der Grad der Vollständigkeit des Zielsystems nach Durchführung der Methode als höher erachtet, was den Schluss zulässt, dass Definitionslücken reduziert wurden. Weiter wird nach der Methodendurchführung die Sicherheit, die aus Kunden und Anwendersicht richtigen Zielsystemelemente erhoben zu haben, auch höher wahrgenommen. Folglich kann der Schluss gezogen werden, dass Wissenslücken reduziert wurden.

Um weiterführende Erkenntnisse zu erlangen wird in nachfolgenden Arbeiten tiefergehend die Auswirkung auf die vorliegende Unsicherheit analysiert. Dazu soll neben

Umgang mit Marktunsicherheiten in der Zielsystementwicklung: 
der subjektiven Einschätzung hinsichtlich dieser auch die gezielte Validierung von identifizierten Zielsystemelementen durch MVPs durchgeführt werden. Darüber hinaus soll der Einfluss der Integration von Kunden und Anwender statt Mitarbeitern in den Prozess der Konkretisierung des initialen Zielsystems untersucht werden. Dieser soll dazu im Rahmen einer Studie mit Test- und Kontrollgruppe ermittelt werden.

\section{Literaturverzeichnis}

Albers, A. (2010): Five Hypotheses about Engineering Processes and their Consequences. In: Horváth I. \& Mandorli, F., Rusák, Z. (Hrsg.), 8th International Symposium on Tools and Methods of Cometitive Engineering.

Albers, A., Bursac, N. \& Wintergerst, E. (2015): Produktgenerationsentwicklung - Bedeutung und Herausforderungen aus einer entwicklungsmethodischen Perspektive. In: Binz, H., Bertsche, B. \& Bauer, W. (Hrsg.), Proceedings Stuttgarter Symposium für Produktentwicklung 2015 SSP 2015. Stuttgart: Fraunhofer Verlag.

Albers, A., Ebel, B. \& Lohmeyer, Q. (2012): Systems of Objectives in Complex Product Development. In: Horváth, I., Albers, A., Behrendt, M. \& Rusák, Z. (Hrsg.), Proceedings of TMCE 2012.

Albers, A., Heimicke, J., Walter, B., Basedow, G. N., Reiß, N., Heitger, N., Ott, S. \& Bursac, N. (2018): Product Profiles: Modelling customer benefits as a foundation to bring inventions to innovations. In: Procedia CIRP, 70, S. 253-258.

https://doi.org/10.1016/j.procir.2018.02.044

Albers, A., Rapp, S., Spadinger, M., Richter, T., Birk, C., Marthaler, F., Heimicke, J., Kurtz, V. \& Wessels, H. (2018): The Reference System in the Model of PGE: Proposing a Generalized Description of Reference Products and their Interrelations. In: Proceedings of the Design Society: International Conference on Engineering Design 2018, 1(1), S. 1693-1702. https://doi.org/10.1017/dsi.2019.175

Albers, A., Zimmermann, V., Marthaler, F., Bursac, N., Duehr, K. \& Spadinger, M. (2021): Selection of Reference System Elements in the Model of PGE: Method for the Integration of Customer and User Satisfaction in Product Planning. In: Proceedings of the Design Society: International Conference on Engineering Design 2021, in Druck.

Bik, N., Lucassen, G. \& Brinkkemper S. (2017): A Reference Method for User Story Requirements in Agile Systems Development. In: IEEE 25th International Requirements Engineering Conference Workshops (REW), S. 292-298.

https://doi.org/10.1109/REW.2017.8

Blessing, L. T.M. \& Chakrabarti, A. (2009): DRM, a Design Research Methodology. London: Springer. https://doi.org/10.1007/978-1-84882-587-1

Dieste, 0., Juristo, N. \& Shull, F. (2008): Understanding the Customer: What Do We Know about Requirements Elicitation? In: IEEE Software 25(2), S. 11-13. https://doi.org/10.1109/MS.2008.53

Herstatt, C. \& von Hippel, E. (1992): From experience: Developing new product concepts via the lead user method: A case study in a "low-tech" field. In: Journal of Product Innovation Management 9(3), S. 213-221. https://doi.org/10.1016/07376782(92)90031-7.

Ili, S., Albers, A. \& Miller, S. (2010): Open innovation in the automotive industry. In: R\&D Management, 40(3), S. 246-255. 
International Council on Systems Engineering - INCOSE (2015): Systems engineering handbook. A guide for system life cycle processes and activities. Hoboken, NJ: Wiley.

Logue, K., \& McDaid, K. (2008): Handling Uncertainty in Agile Requirement Prioritization and Scheduling Using Statistical Simulation. In: Melnik, G. (Hrsg.), Conference Agile, 2008. AGILE'08; August 4 - 8, 2008, Toronto, Ontario, Canada (S. 7382). Los Alamitos, Calif.: IEEE Computer Soc.

McManus, H. \& Hastings, D. (2004) A framework for understanding uncertainty and its mitigation and exploitation in complex systems. In: Engineering Management Review, 34(3). https://doi.org/10.1109/EMR.2006.261384

Nielsen, L. (2018) Design Personas - New ways, new contexts. In: Persona Studies, 4(2), S. 1-4. https://doi.org/10.1109/EMR.2006.261384

Pruitt, J. \& Grudin, J. (2003): Personas: Practice and Theory. In: Arnowitz, J. (Hrsg.), Proceedings of the 2003 conference on Designing for user experiences. New York, NY: ACM.

Verworn, B. \& Herstatt, C. (2007): Strukturierung und Gestaltung der frühen Phasen des Innovationsprozesses. (Bd. 24) Wiesbaden: Betriebswirtschaftlicher Verlag Dr. Th. Gabler | GWW Fachverlage GmbH. https://doi.org/10.1007/978-3-83499293-2_6

Zimmermann, V., Heimicke, J., Bohn, R., Schuhmacher, L., Trost, S. \& Albers, A. (2020): Herausforderungen des agilen und sequentiellen Zielsystem- und Anforderungsmanagements. In: Corves, B., Gericke, K., Grote, K.-H., Lohrengel, A., Löwer, M., Nagarajah, A. (Hrsg.), 18. Gemeinsames Kolloquium Konstruktionstechnik 2020. Nachhaltige Produktentwicklung (S. 245 258). Duisburg-Essen: DuEPublico.

Zimmermann, V., Prinz, R. \& Albers, A. (2020): Methode zur gezielten Identifikation von Marktunsicherheiten zur Unterstützung der Zielsystementstehung Proceedings of the 31st Symposium Design for X (DFX2020) (S. 209-218). The Design Society. https://doi.org/10.35199/dfx2020.22

\section{Kontakt}

Valentin Zimmermann, M. Sc.

Christoph Kempf, M. Sc.

Leo Hartmann, M. Sc.

Univ.-Prof. Dr.-Ing. Dr. h. c. Albert Albers

Institut für Produktentwicklung (IPEK) am Karlsruher Institut für Technologie (KIT)

Kaiserstraße 10

76131 Karlsruhe

http://www.ipek.kit.edu

Dr.-Ing. Nikola Bursac

TRUMPF Werkzeugmaschinen GmbH + Co. KG

Johann-Maus-Straße 2

71254 Ditzingen

https://www.trumpf.com/de_DE 